\title{
Thinking on Engineering Methodology and Innovation for Equipment Health Management
}

\author{
Guojun Qin ${ }^{1,2}$ Zhe Cheng ${ }^{1,2}$ Junyao $\mathrm{Li}^{1,2}$ Niaoqing $\mathrm{Hu}^{1,2}$ \\ ${ }^{1}$ Laboratory of Science and Technology on Integrated Logistics Support, National Uni- \\ versity of Defense Technology, 410073, Changsha, China \\ ${ }^{2}$ College of Mechatronic Engineering and Automation, National University of Defense \\ Technology, 410073, Changsha, China
}

\begin{abstract}
As a system engineering, equipment health management connects with all departments on the design, application and maintenance, and run through each chain in life cycle of the object equipment. It is one of the hot points in recent years in engineering study and has abundant theoretical and technological connotation. In this paper, the engineering methodology for equipment health management, including philosophical basis, general methods, as well as innovation force and approaches, are studied. Aiming at the training of innovation ability at the related technical direction, the guiding function of health management engineering methodology on innovation studies is expounded trough the aspects of topic title and scientific research .
\end{abstract}

Keywords: engineering methodology; health management; equipment; innovation ability

\section{Introduction}

After the concept of failure prevention was put forward in the 1960s, with the increasing of the equipment complexity and the requirements on reliability, safety, etc, health management engineering (EHM) is improving day by day. As an important direction of engineering subjects, it refers to the integration of theories and technologies about material, mechanics, electronics, control, instrument, computer, and other subjects. At present, with the development of EHM from failure maintenance and preventive maintenance to damage mechanism and evolution analysis, embedded/built in test, early fault detection and localization, fault prediction and autonomous maintenance. The researchers have to face many new challenges such as how to combine the latest theory in related discipline with EHM, how to solve the scientific and technical problems innovatively in engineering practice, etc. As there are a lot of objects and specific technologies related in EHM engineering, the nature in the study of EHM's complex details should be revealed, which is not only a technology innovation point but also a philosophical point.

Scientific methodology is a general theory about science study methods, whose research is almost as old as science itself. In the history of science study, the successful scientists, from Aristotle, Newton, Popper, Einstein to Tsien ${ }^{[1,2]}$, were all applying the scientific methodology in their researches initiatively or involuntarily, especially 
since the 20th century. Therefore, the study of EHM engineering methodology and its application to the research practice must have important guiding significance on improving relevant innovation ability.

\section{EHM engineering methodology}

EHM engineering methodology, as the combination of materialist dialectics and EHM engineering, refers to the general methods for EHM research, the trend and direction of related methods development, and the relationship between all kinds of research methods.

\subsection{Philosophical basis}

The philosophical basis of EHM engineering methodology can be summarized into four levels — "the world is material, all materials are in motion, all motions are in regular, all rules can be recognized and used".

First of all, in EHM engineering, once the equipment or its component manufactured, their own life cycle will start. Its health states never depend on the researcher/user's perception. People can test, analysis, control, adjustment and do other operations, but can't change its basic attributes of "matter".

Secondly, no matter whether the equipment runs or not, its health state changes constantly under the action of itself and outside conditions. People can adjust the procedure by maintenance, operation and environment changing, but can never stop it.

Thirdly, although the starting point of the health state can be adjusted through maintenance, repair etc, the deterioration trend in the whole life cycle of equipment is constant. All failure and damage starts from the microscopic damage, gradually extends to components and subsystem, until the system failures. Both the wastage fault and sudden failure follow the same rules.

Fourthly, although not all objective laws of health degradation can be recognized accurately at present for the limitation of science development, it should firmly believe that new objective laws, methods and means must be found.

Finally, people are not powerless in equipment health degeneration. By mastering the regularity, the reality degradation can be understood accurately, and the degradation process can be predicted and controlled. The catastrophic accidents can be prevented.

\subsection{General method}

In the process of health degradation, the degradation state, reason and performance are variable in complex system. Therefore, the general methods of EHM engineering have its own particularity, it mainly including:

(1) Combination of universality and individuality

Specific equipment which needs EHM has often a certain commonality with other same type ones. Even for large complex equipment, the degradation process of its main subsystem or components is often similar with the same type ones in other equipments. EHM should make full use of these universalities, which can be obtained from theoretical analysis, system simulation, fault embedding experiments and historical statistics of similar systems. At the same time, modern health management pays more attention to give a private solution for equipments. For the difference of health starting point, operating environment, only considering the individuality sufficiently, can we obtain the best EHM scheme.

(2) Combination of deductive and inductive

For the main goal of equipment design and manufacture is how to realize 
the normal performance, large number of abnormal states and degradation process are often not considered sufficiently. But as to the EHM, the fault or health degradation must be considered firstly. So, deductive methods, such as modeling and simulation, are needed to obtain the features of abnormal states as comprehensive as possible, and change "black box problem" to "white box" or "gray box" problem. At the same time, the simulation conditions are impossible to exactly the same with real application. For long life equipments, inductive methods can be adopted to mine information. So, the combination of deductive and inductive is an important method to solve the problem of EHM.

(3) Unity of monitoring, diagnosis, prognosis, and life management

"Condition monitoring-fault diagnosis -prognosis -life management" is the spiral development process of EHM, in which condition monitoring determine the states, diagnosis locate fault, prognostics predict when and which part is going to fault/failure. Condition monitoring is the basis of diagnosis and prognostics, diagnosis and prognostics provide guidance for life management, and life management formulates reference for monitoring strategy.

(4) Progress of practice, theory and re-practice

As a subject for application, EHM engineering needs to solve the problems from the practice. After common problems are repeated and analyzed, the algorithms are designed, integrated and applied to real practice. If the research and technology can't solve the problem very well, new study is needed.

\section{Thinking on innovation of EHM engineering}

Innovation ability refers to using accumulated knowledge and experience to generate new knowledge, ideas, methods and achievements through thinking process and innovation ${ }^{[3]}$. To improve the innovative ability of EHM engineering, the methodology should be excavated and implied comprehensively during the research stage.

\subsection{Innovative force and approach}

The innovative force of EHM engineering is a moment adhesion to the traditional skepticism and critical spirit. As other disciplines, the initial understanding in the research of EHM engineering may be not correct. It is likely occurred deviation due to the limitation of damage dynamics, test technology, signal processing method at the time. At the same time, for the EHM objects went into microscopic, cosmic and high speed field, it is often found that the traditional sense of theoretical system, solution method and research ideas need to be further revision and improvement. All these need keep skepticism and critical spirit of tradition during the study and practice. Using the latest scientific research auxiliary means to test the traditional theory or method, and constantly add sound to form more scientific and perfect theory system.

What EHM engineering has always been traded using the bold exploration is the innovative way of thinking route, whose obvious characteristic is the integrated development trend leaded by highly differentiation and integration. A growing number of edge and comprehensive disciplines has promoted the mutual infiltration between disciplines, and communicate the separated domain with each other. Therefore, all kinds of new theory and methods need to be investigated using creative exploration to find. 


\subsection{Direction of the research topic selection}

Scientific research process generally includes five steps as "subject selectionplan schedule - work and test - paper or report writing". As to the researchers, choosing subject is the precondition of research and achievements. During the subject selection, following points should be mainly considered:

(1) Fusion of systemic thinking and focused thinking

System features of EHM and the common feature of equipments should be considered firstly. After the comprehensive understanding of the field and determining of main direction, certain key scientific or technical problems should be focused on, and the possible technological approach should be considered.

(2) Fusion of innovative thinking and traditional thinking

Innovative thinking includes the methods of original innovation, integrated innovation and study-based innovation. The research topic should be a scientific or engineering problem not completely resolved and has dawn to achieve. At the same time, the dialectical unification of innovative thinking and traditional thinking need to be followed. The researchers should neither go on in the same old rut or being bound by traditional theory and methods, nor blindly pursue innovation, breaking away from EHM requirements, feasibility and targets.

(3) Fusion of forward-looking thinking and feasibility thinking

In topic selection, the researcher need to pay attention to all relevant researches, master the key technologies from the frontiers, and use forward-looking thinking to find possible developing direction and applications of key technology. At the same time, their knowledge and engineering experience, the development on related disciplines and the conditions should be considered to avoid the choosing of a topic without feasibility.

\subsection{Guidance for the research phase}

During the research phase, EHM engineering methodology needs to be brought in for guiding the topic. In general, it reflected in the thinking implementation of critical, wholeness, intersectional, repetitive, etc.

(1) To carry out critical thinking

Critical thinking is the authenticity, accuracy and the value of knowledge in personal judgment ${ }^{[4]}$. Creation begins from doubt, thinking and criticism. In the field of EHM project, there is a lot of empirical formula and linearization method. Therefore, research should not only simply to paraphrase, but using the critical way of thinking to analyze whether the application fields of the empirical formula have changed, and whether linearization has important influence on the result. Based on the actual research object and the test results, putting forward bold question and criticize of the traditional experience conclusion and forming the innovation.

(2) Carry out wholeness thinking

Scientific methodology emphasizes the dialectic view of "the whole is greater than the sum of the parts". In the field of EHM engineering, the traditional thinking is to separate the system into multiple parts, diagnose and forecast respectively, then draw a conclusion after comprehensive. The new methods are gradually to break through the traditional constraints. It carries out the holistic thinking and makes all parts information as a whole analysis and induction. It uses information fusion method, makes up for the inadequacy of their methods and improves accuracy and reliability.

(3) Carry out intersectional thinking

Intersectional thinking breaks out the strict boundaries between EHM and other 
areas. In traditional health degradation feature extraction of rotating machinery, only speed, temperature and vibration frequency characteristic could not completely meet all actual needs. With the introduction of machine learning theory, immune method, finite element analysis, etc., the actual problem has been solving from information aspect. It obtains good effect and embodies the application of its thinking results.

(4) To carry out repetitive thinking

Any scientific research process is not plain sailing, nor to that on EHM. Lots of differences may create in theory calculation, test and application, and it also could not completely quite be same. So, the scientific research of repeatability needs to be fully understood. When setbacks appear, we must be good at reflecting on their own practice, and summarizing the lessons in failures. At the same time, we should fully realize that EHM engineering itself is a process need to be maturated constantly, and improved in a long-term accumulation and good tempers.

\section{Conclusion}

Although the relevant technology on the equipment failure prevention has been studied for more than 40 years, as a cross discipline put forward and generally expected in recent 10 years, EHM need to introduce the corresponding methodology in current research background, and guide the perfection and extension in the new field. In this paper, induction and synthesis was summarized for equipment health management project methodology. At the same time, combining with the research topic selection as well as subject study process, a specific guidance was put forward.

\section{Acknowledgement}

The authors awfully acknowledge the financial support from Hunan graduate education research project of China (grant No.12B03) and education research project of National University of Defense Technology (grant No. yjsy 20102005).

\section{References}

[1] C. Hu and J. Wang, "On Scientific Innovative Methodology Principles of Einstain and Bohr", J. Jinggangshan Normal College, 24(4), pp. 73-76, 2003

[2] Y. Jiang. "Enlightenment from the Foundation of Engineering Cybernetics by Tsien Hsue-sen", Studies in Science of Science, 24(4), pp. 517523, 2006

[3] Q. Yue. “Understanding and Reflection on Graduate Education Inovation Project", J. North China Institute of Science and Technology, 4(2), pp.122-124, 2007

[4] G. T. "Ten Experience from Graduate Education", Academic Degrees \& Graduate Education, 4, pp. 5-8, 2007 